\title{
Gluconobacter dominates the gut microbiome of the Asian palm civet Paradoxurus hermaphroditus that produces kopi luwak
}

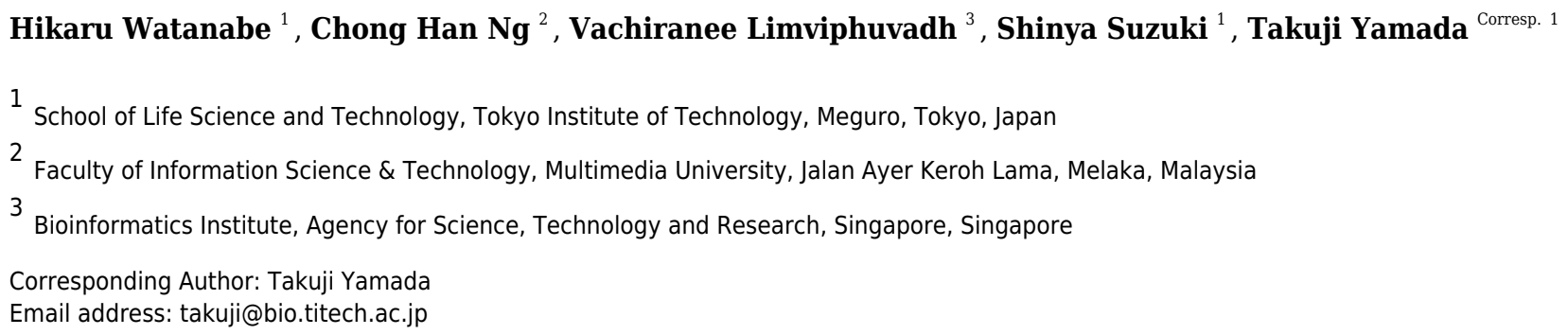

Coffee beans derived from feces of the civet cat are used to brew coffee known as kopi luwak (the Indonesian words for coffee and palm civet, respectively), which is one of the most expensive coffees in the world owing to its limited supply and strong market demand. Recent metabolomics studies have revealed that kopi luwak metabolites differ from metabolites found in other coffee beans. To produce kopi luwak, coffee beans are first eaten by civet cats. It has been proposed that fermentation inside the civet cat digestive tract may contribute to the distinctively smooth flavor of kopi luwak, but the biological basis has not been determined. Therefore, we characterized the microbiome of civet cat feces using 16S rRNA gene sequences to determine the bacterial taxa that may influence fermentation processes related to kopi luwak. Moreover, we compared this fecal microbiome with that of 14 other animals, revealing that Gluconobacter is a genus that is uniquely found in feces of the civet cat. We also found that Gluconobacter species have a large number of cell motility genes, which may encode flagellar proteins allowing colonization of the civet gut. In addition, genes encoding enzymes involved in the metabolism of hydrogen sulfide and sulfur-containing amino acids were over-represented in Gluconobacter. These genes may contribute to the fermentation of coffee beans in the digestive tract of civet cats. 


\section{Manuscript Title}

2 Gluconobacter dominates the gut microbiome of the Asian palm civet Paradoxurus

3 hermaphroditus that produces kopi luwak

4

5 Hikaru Watanabe ${ }^{1}$, Chong Han $\mathrm{Ng}^{2}$, Vachiranee Limviphuvadh ${ }^{3}$, Shinya Suzuki ${ }^{1}$, Takuji

6 Yamada $^{1}$

7

8

$9{ }^{1}$ School of Life Science and Technology, Tokyo Institute of Technology, Meguro, Tokyo, Japan

$10{ }^{2}$ Faculty of Information Science \& Technology, Multimedia University, Jalan Ayer Keroh

11 Lama, 75450 Bukit Beruang, Melaka, Malaysia

$12{ }^{3}$ Bioinformatics Institute, Agency for Science, Technology and Research (A*STAR), 30

13 Biopolis Street, \#07-01 Matrix, 138671, Singapore

14

15 Corresponding Author:

16 Takuji Yamada ${ }^{1}$

17 School of Life Science and Technology, Tokyo Institute of Technology, M6-4, 2-12-1

18 Ookayama, Meguro-ku, Tokyo, Japan

19 Email address: takuji@bio.titech.ac.jp

20

\section{Abstract}

22 Coffee beans derived from feces of the civet cat are used to brew coffee known as kopi luwak (the Indonesian words for coffee and palm civet, respectively), which is one of the most expensive coffees in the world owing to its limited supply and strong market demand. Recent metabolomics studies have revealed that kopi luwak metabolites differ from metabolites found in other coffee beans. To produce kopi luwak, coffee beans are first eaten by civet cats. It has been proposed that fermentation inside the civet cat digestive tract may contribute to the distinctively smooth flavor of kopi luwak, but the biological basis has not been determined. Therefore, we characterized the microbiome of civet cat feces using $16 \mathrm{~S}$ rRNA gene sequences to determine the bacterial taxa that may influence fermentation processes related to kopi luwak. Moreover, we compared this fecal 
32 found in feces of the civet cat. We also found that Gluconobacter species have a large number of 33 cell motility genes, which may encode flagellar proteins allowing colonization of the civet gut. In 34 addition, genes encoding enzymes involved in the metabolism of hydrogen sulfide and sulfur35 containing amino acids were over-represented in Gluconobacter. These genes may contribute to 36 the fermentation of coffee beans in the digestive tract of civet cats.

\section{Introduction}

39 Coffee beverages have become the most popular staple drinks in many countries. Among 40 different coffee varieties, kopi luwak is one of the most famous, in which the beans are collected 41 from feces of the civet cat (Paradoxurus hermaphroditus). In many countries of Southeast Asia, 42 including Philippines, Malaysia, and Indonesia, wild civet cats eat the beans produced by coffee trees (Patou et al., 2010). Once eaten, the outer layers of the beans are digested inside the gastrointestinal tract. When partially digested coffee beans are excreted in the feces, they are collected, cleaned, dried, and roasted. The roasted beans are then marketed as an expensive commodity (Jumhawan et al., 2016). It has been shown that the chemical and physical properties

47 of kopi luwak beans are different from those of other coffees (Marcone, 2004). Indeed, a metabolomics approach showed that kopi luwak beans contain higher malic acid and citric acid contents and inositol-pyroglutamic acid ratios (Jumhawan et al., 2013). In another study, it has been shown that kopi luwak has lower levels of proteins but higher levels of lipids and carbohydrates than other coffees (Marcone, 2004).

Although the molecular mechanisms for these differences remain unknown, it is possible that the fermentation process inside the civet gut may influence the content of the kopi luwak metabolites. The unique gut microbiome of the civet cat might contribute to the characteristic physical properties and metabolite content of kopi luwak beans. A recent isolation and cultivation approach identified the bacterial species in the civet cat gastrointestinal tract, 
57 including Bacillus, Pseudomonas, Pantoea, Escherichia, Lactobacillus, Ochrobactrum, and

58 Kocuria (Suhandono et al., 2016). However, the study was unable to define the entire

59 microbiome because some of the gut bacteria were unculturable (de Jager \& Siezen, 2011). To

60 address this shortcoming, we assessed the gut microbiome of the civet cat to identify the

61 bacterial species that may contribute to the fermentation of kopi luwak beans using the $16 \mathrm{~S}$

62 rRNA gene sequence. This approach allows us to detect unculturable bacteria. We collected

63 three samples of civet cat feces from a Malaysian coffee farm. To characterize the civet cat fecal

64 microbiome, we used the 16S rRNA gene sequence to determine the bacterial taxonomy. Our

65 microbiome analysis of the 16S rRNA gene sequence revealed an abundance of Gluconobacter

66 species. Our comparative genomics between Gluconobacter species, which are most similar to

67 the Gluconobacter of the civet cat gut microbiome, revealed that the over-represented genes of

68 certain sulfur metabolic pathways, such as sulfate reduction or cysteine and methionine

69 metabolism, were also among the top-ranked modules/pathways. These pathways may contribute

70 to the fermentation of kopi luwak beans in the gut.

71

\section{Materials and Methods}

73 Collection of fecal samples and DNA extraction

74 Three fresh fecal samples from wild civets were collected in the morning on June 20, 2015

75 from a coffee plantation (GPS coordinate: latitude: 1.7495934, longitude:103.387647) in Johor,

76 Malaysia. The samples were kept at $4^{\circ} \mathrm{C}$ for $\leq 2 \mathrm{~h}$ before being used for DNA extraction. To

77 improve the DNA yield during extraction, $\sim 0.2 \mathrm{~g}$ of each fecal sample was first incubated in 200

$78 \mu \mathrm{l}$ of suspension buffer ( $10 \mathrm{mM}$ Tris- $\mathrm{HCl}, \mathrm{pH}$ 8.0, $0.1 \mathrm{mM}$ EDTA) containing $2 \mathrm{mg} / \mathrm{ml} \mathrm{lysozyme}$ 
79 (Sigma Aldrich, St. Louis, MO, USA) at $37^{\circ} \mathrm{C}$ for $30 \mathrm{~min}$ to remove the bacterial cell wall. The 80 DNA was extracted from feces using the QIAmp® DNA stool kit (Qiagen, Hilden, Germany).

81 To digest RNA, RNase A (Sigma Aldrich, St. Louis, MO, USA) was added (final concentration 82 of $0.1 \mathrm{mg} / \mathrm{ml}$ ) to buffer ASL from the stool kit. The quantity and quality of genomic DNA were 83 estimated using a NanoDrop ${ }^{\text {TM }}$ 2000/2000c spectrophotometer (Thermo Fisher Scientific,

84 Waltham, MA, USA).

PCR amplification, sequencing, and analysis of the $16 \mathrm{~S} r R N A$ gene

A fragment of the 16S rRNA gene was amplified using the prokaryotic universal primer set 27F (5'-AGAGTTTGATCCTGGCTCAG-3') and 338R (5'-TGCTGCCTCCCGTAGGAGT-3')

and Ex Taq DNA polymerase, hot-start version (Takara Bio, Shiga, Japan). PCR was carried out in $50-\mu 1$ reaction volumes containing forward and reverse primers ( $1 \mu \mathrm{M}$ each), $20 \mu$ of template DNA, $5 \mu \mathrm{l}$ of $10 \times$ Ex Taq Buffer, and $4 \mu \mathrm{l}$ of dNTP mix from the Ex Taq kit. The PCR program consisted of $95^{\circ} \mathrm{C}$ for $5 \mathrm{~min}$ followed by 31 cycles of $95^{\circ} \mathrm{C}$ for $30 \mathrm{~s}, 53^{\circ} \mathrm{C}$ for $30 \mathrm{~s}$, and $72^{\circ} \mathrm{C}$ for $30 \mathrm{~s}$, and a final extension at $72^{\circ} \mathrm{C}$ for $3 \mathrm{~min}$. The PCR products were individually concentrated and purified using a 2\% E-Gel SizeSelect agarose gel (Thermo Fisher Scientific, Waltham, MA, USA), quantified using the Quant-iT dsDNA HS Assay kit (Thermo Fisher Scientific, Waltham, MA, USA), and qualified using the High Sensitivity DNA kit (Agilent Technologies, Santa

97 Clara, CA, USA). Then, we checked a negative-control sample, in which PCR produced no 98 visible bands on an agarose gel. Sequencing was carried out with the Ion PGM Sequencing 400 99 kit (Thermo Fisher Scientific, Waltham, MA, USA). Raw data for 16S rRNA gene sequences 100 were analyzed with the VITCOMIC2 web application (http://vitcomic.org/) to derive the genus composition with the option 'Conduct 16S rRNA gene Copy number normalization? - No'. 
102 VITCOMIC2 can estimate microbial community composition based on the sequence data for the

103 16S rRNA gene obtained from both metagenomic shotgun and amplicon sequencing (Mori et al.,

104 2018). The sequence data are available in the DDBJ DRA database

105 (https://www.ddbj.nig.ac.jp/dra/index-e.html) under accession DRA006640.

106

107 16S rRNA gene sequence-based phylogenetic analysis

108 The 16S rRNA gene sequences from bacteria in civet cat feces were checked using Trimmomatic 109 version 0.33 with parameters 'SE LEADING:17 TRAILING:17 AVGQUAL:25 MINLEN:200'

110 to filter out low-quality sequences (Bolger, Lohse \& Usadel, 2014). The three most abundant

111 Gluconobacter sequences (termed civet cat004:Uniq1, civet cat005:Uniq1, civet cat006:Uniq1)

112 were retrieved from each sample using the fastx unique command implemented in USEARCH

113 version 10.0.240 (Edgar, 2010). To compare our Gluconobacter 16S rRNA gene sequences with

114 those of other Gluconobacter species, we collected 16S rRNA gene sequences of Gluconobacter

115 and Acetobacter from the SILVA Living Tree Project version 128 (Yilmaz et al., 2014). The

116 sequences were aligned with MAFFT version 7.313 (Nakamura et al., 2018) and used as input to

117 construct a phylogenetic tree using the neighbor-joining method with a Kimura 2-parameter

118 model of nucleotide substitution using MEGA7 (Kumar, Stecher \& Tamura, 2016). Acetobacter

119 was used as an outgroup as the most similar taxonomic group to Gluconobacter species.

120

121 Collection of animal fecal $16 S$ rRNA gene sequence data from other studies

122 Data for the bacterial genera identified in feces of various animals were collected from 123 MicrobeDB.jp (MicrobeDB.jp Project Team, 2017). MicrobeDB.jp is a public database that stores 
124 the taxonomic composition data computed by VITCOMIC2 for most 16S rRNA gene sequences

125 in the International Nucleotide Sequence Database Collaboration-Sequence Read Archive 126 (INSDC-SRA), with integrated sampling site information such as environment and host categories.

127 After downloading all the taxonomic composition data, all data with alignment counts less than 1281,000 were filtered out. We listed the number of 16S rRNA gene sequence samples used in a 129 comparative analysis after quality control of the raw sequence data (Table 1). Next, we converted 130 alignment counts to mean relative abundance for each sample. After selecting samples that had an 131 annotation of feces (MEO_0000054) by Metagenome and Microbes Environmental Ontology 132 (MEO), we grouped samples into host categories and calculated their average relative abundances 133 to serve as a reference microbiome composition for the gut of all animals in the analysis 134 (MicrobeDB.jp Project Team, 2017).

Comparative genomics of the major microorganisms in feces from civet cat and other animals

137 To provide a plausible explanation for the known metabolite profiles of kopi luwak, we 138 compared the major bacterial species in civet cat feces ( $G$. frateurii and G. japonicus) with the 139 top nine dominant bacterial genera in feces from other animals (Bacteroides, Prevotella, 140 Barnesiella, Lactobacillus, Oscillibacter, Citrobacter, Streptococcus, Faecalibacterium, and 141 Enterococcus). We downloaded complete amino acid sequences for each bacterial species from 142 the National Center for Biotechnology Information (NCBI) RefSeq collection (Table S1) and 143 annotated them with the reference prokaryote Kyoto Encyclopedia of Genes and Genomes 144 (KEGG) orthology (KO) amino acid sequences (KEGG FTP Release 82.0) using DIAMOND 145 version 0.9.3 with the parameter 'blastp --max-target-seqs 5 --evalue 1e-2 --id 70 --min-score 40' 146 (Buchfink, Xie \& Huson, 2015). We used the top-hit results with e-values less than 1e-8 as the 
$147 \mathrm{KO}$ annotation for the amino acid sequences.

148 To compare KO terms, we calculated the median number of paralogs for each KO in each

149 bacterial genus, including Gluconobacter from the NCBI RefSeq collection (Table S1). For the

150 Gluconobacter genus, we used G. frateurii and G. japonicus because these two species are most

151 closely related to the Gluconobacter species of the civet cat fecal microbiome (Table S2). To

152 determine the $\mathrm{KO}(\mathrm{s})$ that was over-represented in Gluconobacter but not in major fecal microbial 153 genera of other animals, we calculated the $\mathrm{KO}_{(\mathrm{i}, \text { ratio })}$ as follows.

154

155

156

157

158

159

160

161

162

163

164

165

166

167

168

$$
\mathrm{KO}_{(\mathrm{i}, \text { ratio })}=\frac{K O_{(\mathrm{i}, \text { Gluconobacter })}}{\mathrm{KO}}
$$

Here, the $K O_{(\mathrm{i}, \text { Gluconobacter) }}$ is calculated as the median for the number of $\mathrm{KO}_{\mathrm{i}}$ homologs in each Gluconobacter strain. Conversely, $K O_{(\mathrm{i}, \text { major fecal microbial genera) }}$ is the mean of the median for the the number of $\mathrm{KO}_{\mathrm{i}}$ homologs in the top nine major bacterial genera in other animal feces (see Supplemental Table 2). To estimate the over-represented functional units (KEGG module / KEGG pathway) in Gluconobacter (G. frateurii and G. japonicus), we performed Fisher's exact test for each functional unit based on the number of over-represented KOs belonging to each functional unit in Gluconobacter. The $\mathrm{KO}_{\text {ratio }_{i}}$ was converted to the $\log _{2}$ fold-change. We calculated A) the number of KOs in a specific functional unit i, B) the number of KOs out of the functional unit $i, C$ ) the number of KOs with a $\log _{2}$ fold-change greater than 1 in specific functional unit $\mathrm{i}$, and D) the number of KOs with a $\log _{2}$ fold-change greater than 1 in functional unit $i$. These four types of numbers for $\mathrm{KO}$ were used to perform Fisher's exact test with R version 3.3.2 (R Core Team; Fisher, 1962). 


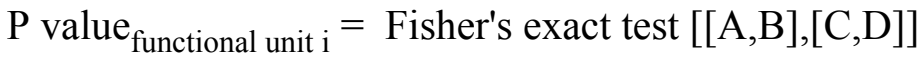

171

172 For a $P$ value $\leq 0.05$ with Fisher's exact test, the generated functional unit i can be considered a 173 special functional unit of G. frateurii and G. japonicus.

174 To identify functional units in G. frateurii and G. japonicus with $\log _{2}$ fold-changes $>1$

175 relative to the fecal microbes from other animals, we mapped each functional unit ( $P$ value $176 \leq 0.05$ ) and the positive ratio of $G$. frateurii and G. japonicus for each KO using the FuncTree2

177 web service (https://bioviz.tokyo/functree2/) (Uchiyama et al., 2015). This web service is a

178 hierarchical visualization tool for KEGG pathways/modules/KO.

179

180

Results

181

182

183

184

185

186

187

188

189

190

191

\section{Comparison of fecal microbial communities between civet cat and other animals}

We analyzed the 16S rRNA gene sequences of microbes in each of three feces samples obtained from three different civet cats, which yielded 28,120,904 raw reads. Among all samples, the five most average relative abundant genera were Gluconobacter (66\%), Citrobacter (14\%), Acetobacter (7\%), Enterobacter (2\%), and Clostridium XI (1\%) (Table S3). To determine the bacterial genus that is predominant in the civet cat fecal microbiome, we compared the civet cat fecal 16S rRNA gene sequencing results with those obtained from the fecal microbiome of other animals. The data for the genus composition of the fecal microbiome of 14 animals were obtained from MicrobeDB.jp (MicrobeDB.jp Project Team, 2017)(http://microbedb.jp).

Microbial composition data derived from the same computational pipeline were used for the civet cat fecal microbiome 16S rRNA gene sequence analysis. Among all fecal samples from all 
192 animals, the largest sample size was that of human feces $(n=3,003)$, with the second largest

193 being mouse feces $(n=1,346)$. Most of the human feces samples have been acquired previously

194 for large-scale projects such as the Human Microbiome Project (Turnbaugh et al., 2007) and the

195 Metagenomics of the Human Intestinal Tract project (Ehrlich, Dusko Ehrlich \& The MetaHIT

196 Consortium, 2011) (Table 1).

197 We determined the microbial genus composition of feces for each animal species by

198 averaging the relative abundance among all fecal samples for each animal. A comparison of

199 these derived compositions with that determined for civet cat feces revealed a marked difference

200 in bacterial genus composition (Fig. 1 and Table S3). For example, Gluconobacter was found to

201 be the predominant genus, representing $60 \%$ of the bacterial abundance in all civet cat samples.

202 By contrast, Gluconobacter was rarely detected in the fecal microbiomes of the 14 animals used

203 in the other studies. Therefore, civet cat apparently has a unique fecal microbiome compared

204 with other animals.

205

206

Phylogenetic analysis of Gluconobacter species in civet cat feces

207

To determine the Gluconobacter species that are most common in the civet cat fecal

208

microbiome, we created a phylogenetic tree for Gluconobacter species and Acetobacter strains

209 as the outgroup in civet cat fecal samples (Fig. 2). The tree showed that Gluconobacter

210 comprises two groups, namely group 1 and group 2 . The species in civet cat fecal samples

211 belonged to group 2 (Fig. 2). The tree also showed that the Gluconobacter species in the civet cat

212 fecal microbiome belonged to the group including G. cerinus, G. frateurii, G. japonicus, G.

213 nephelii, G. thailandicus, and G. wancherniae (Fig. 2, Fig. S1). To determine the KO terms for

214 Gluconobacter species, we calculated the KO terms for the amino acid sequences of 39

PeerJ reviewing PDF | (2020:01:45194:1:1:REVIEW 13 Jun 2020) 
215 Gluconobacter strains from the Reference Sequence (RefSeq) database of NCBI. We found that 216 RefSeq genomes for G. nephelii and G. wancherniae were not available in NCBI; hence, these

217 two species were not included in the KO analysis. Next, using hierarchical clustering of the KO

218 terms for each Gluconobacter strain, we showed that strains with similar 16S rRNA gene

219 sequences clustered together, with distinct separations among the clades (Fig. 3). These results

220 demonstrated that the Gluconobacter species of the civet cat fecal microbiome were most likely

221 to be G. frateurii and G. japonicus, and that the KO terms of these two species differed from

222 those of other Gluconobacter species, especially species in Gluconobacter group 1.

223

224

Comparative genomics of genera of the feces microbiome

225

We next compared the KO terms for G. frateurii and G. japonicus, which are the most 226 similar species to the Gluconobacter species of the civet cat fecal microbiome, with the KO 227 terms of the dominant genera in feces of other animals. Other dominant bacterial genera in the 228 feces of other animals were Bacteroides, Prevotella, Barnesiella, Lactobacillus, Oscillibacter, Citrobacter, Streptococcus, Faecalibacterium, and Enterococcus (Fig. 1). This comparative genomics analysis provided a list of candidate microbial genes that may be involved in the fermentation of kopi luwak in the civet cat digestive tract.

To identify the KO terms of each genus, we downloaded the amino acid sequences of RefSeq genomes for these genera (Table S1). These sequences were mapped to prokaryote KO amino acid sequences that are publicly available in the KEGG. The KEGG database contains information about most of the bacterial genome sequences (Kanehisa, 2000). Finally, we calculated the median number of KOs for each genus. Likewise, we calculated the median number of KOs for G. frateurii and G. japonicus because these two species were the 
238 predominant Gluconobacter species of the civet cat fecal microbiome (Fig. 4 and Table S2).

239 Interestingly, certain KO terms were more abundant in G. frateurii and G. japonicus than in

240 other Gluconobacter species or were present in only these two species. We next determined the

241 KO terms that were enriched in G. frateurii and G. japonicus compared with other major genera

242 of the various animal fecal microbiomes in our analysis. We calculated $\log _{2}$ fold-change values,

243 which were derived from the median number of KO terms of G. frateurii and G. japonicus per

244 mean number of $\mathrm{KO}$ terms of other dominant bacterial genera in the feces of other animals; this

245 allowed us to determine enrichment, and we counted the number of $\mathrm{KO}$ terms with $\log _{2}$ fold-

246 change greater than 1.0. These results showed that at least $664 \mathrm{KOs}$ of the 3,623 total had a $\log _{2}$

247 fold-change value $\geq 1.0$ (Table S4). To minimize false-positive results, we performed Fisher's

248 exact test for each KEGG module and pathway (see Methods). The following pathways and

249 modules were found to be more abundant in G. frateurii and G. japonicus than in other animal

250 fecal microbes: porphyrin and chlorophyll metabolism (map00860); cobalamin biosynthesis,

251 cobinamide $=>$ cobalamin (M00122); flagellar assembly (map02040); methionine salvage

252 pathway (M00034); histidine biosynthesis, PRPP => histidine (M00026); assimilatory sulfate

253 reduction, sulfate $=>\mathrm{H}_{2} \mathrm{~S}$ (M00176); cysteine and methionine metabolism (map00270) (Fig. 5).

254 Moreover, we analyzed over-represented KO terms of the TCA cycle (map00020) in G. frateurii

255 and G. japonicus because a previous study demonstrated that the levels of malate and citrate

256 produced from the TCA cycle are higher in kopi luwak beans than other coffee beans (Jumhawan

257 et al., 2016). Although Fisher's exact test could not detect any differences in the KOs for the

258 TCA cycle among different coffee beans (map00020), this test showed that certain KOs of the

259 TCA cycle (map00020) were over-represented in G. frateurii and G. japonicus (Fig. 5 and Table 260 S2). 


\section{Discussion}

263 In this study, we compared the civet cat fecal microbiome with microbiomes that have been

264 established for 14 other animals, including humans. In the civet cat fecal microbiome,

265 Gluconobacter was found to be the most abundant genus. To elucidate the relationship between

266 genetic factors and microbiome composition, we checked public resources concerning the

267 genetic contents of the gut microbiome genera in the family Felidae, which is known to be the

268 closest taxon to the civet cat (Nyakatura \& Bininda-Emonds, 2012). Two studies described the

269 microbiome of the domesticated cat, genus Felidae. However, these studies did not report the

270 presence of Gluconobacter in the samples (Deusch et al., 2014, 2015).

271

272 Another study reported that the human fecal microbiome differs among countries, suggesting

273 that various diets might be able to explain the difference (Moeller et al., 2014; Kartzinel et al.,

274 2019). It has been suggested that different diets may help to explain the uniqueness of the civet

275 cat fecal microbiome, which may be influenced by uncommon foods such as coffee beans. The

276 most important point is that previous studies of the animal fecal microbiome did not detect a

277 microbiome dominated by Gluconobacter.

278

279 Moreover, our phylogenetic analysis demonstrated that Gluconobacter species that are closely to

G. frateurii, G. japonicus, G. nephelii, and G. wancherniae are the most abundant species in the

281 civet cat fecal microbiome. A previous study reported that G. frateurii, G. japonicus are closest

282 to each other (Malimas et al., 2009). Our study suggests that the civet cat fecal microbiome is

283 dominated by Gluconobacter, which was detected in a previous study using general cultivation 
284 methods (Suhandono et al., 2016). In fact, a previous study of the cacao microbiome reported

285 that Gluconobacter species is hardly detected by the cultivation method; however, using

286 metagenomics and shotgun sequencing, the presence of Gluconobacter species was observed

287 (Agyirifo et al., 2019).

288

289 Some bacteria can grow within only a narrow temperature range. To determine the optimum 290 growth temperature of various Gluconobacter species, we retrieved information about the growth 291 temperature of Gluconobacter (NITE, 2018). Most Gluconobacter strains grow at $37^{\circ} \mathrm{C}$, which is 292 the body temperature of the civet cat (Fig. S2). Interestingly, upon their initial sampling from 293 feces, fresh coffee cherries contain a Gluconobacter sp. (De Bruyn et al., 2017). Although we 294 could not analyze the microbiome from fresh coffee cherries prior to digestion, these data 295 support the notion that Gluconobacter of civet cat feces may originate from fresh coffee cherries and that species of this genus can grow at $37^{\circ} \mathrm{C}$ in the gastrointestinal tract of civet cat and may be involved in the fermentation process of kopi luwak. Interestingly, Gluconobacter species are obligate aerobic microorganisms (Barberán et al., 2017); however, a recent study mentioned that oxygen is rich in the small intestine of humans. Likewise, the civet cat intestine may contain some oxygen that could facilitate Gluconobacter fermentation in the civet cat intestine (Berean 301 et al., 2018).

We identified some over-represented KEGG modules or pathways that may influence the 304 physical and chemical properties of kopi luwak. In this study, we focused on KOs that appeared more frequently in $G$. frateurii and G. japonicus than in the major genera of fecal microbiomes 
307 pathways in Gluconobacter. A recent study of fecal microbiomes revealed that bacterial flagellar

308 proteins promote the production of flagellum-specific immunoglobulin derived from the host,

309 and this immunoglobulin type inhibits bacterial motility (Cullender et al., 2013). Although the

310 bacterial flagellum may cause inflammation in the mammalian gut, civet cats may have a tolerant

311 immunity to the flagella of Gluconobacter. In addition, the bacterial flagellum often helps

312 colonize the attachment and invasion of bacteria to the host cell (Johanna Haiko, 2013). Hence,

313 flagellum of Gluconobacter may be related to colonization of them in the civet cat gut.

314 Conversely, the methionine salvage pathway (M00034); histidine biosynthesis, PRPP =>

315 histidine (M00026); assimilatory sulfate reduction, sulfate $=>\mathrm{H}_{2} \mathrm{~S}$ (M00176); and cysteine and

316 methionine metabolism (map00270) were also among the top-ranked modules/pathways. Sulfur-

317 containing compounds are generally volatile and thus can affect the flavor and aroma of many

318 foods (Reineccius, 2005). A previous study has shown that $G$. frateurii can produce $\mathrm{H}_{2} \mathrm{~S}$ from

319 thiosulfate (Lori \& Claus, 1989). Although it has been shown that sulfur-containing compounds

320 contribute to the aroma of coffee (Czerny, Mayer \& Grosch, 1999), it remains to be determined

321 whether the content of sulfur-containing compounds in kopi luwak differ from those of other

322 coffees. In a previous study, Gluconobacter was detected in the fermentation of cacao samples

323 using metagenomics analysis, and this bacteria is implicated in the fermentation process

324 (Agyirifo et al., 2019). Thus, Gluconobacter might contribute to the production of kopi luwak.

325

326 Traditional metabolomics approaches have identified citric acid and malic acid as discriminative

327 metabolites for kopi luwak and other coffees (Jumhawan et al., 2013, 2016). Although the TCA

328 cycle (map00020) was not over-represented and statistically significant in our study, it is one of

329 the pathways involved in the production or consumption of malic acid and citric acid. Consistent 
330 with this phenomenon, we observed some $\mathrm{KO}$ terms with a relatively large number of homologs

331 in Gluconobacter compared with other bacteria in the TCA cycle (Fig. 5). Therefore, the TCA

332 cycle activity in Gluconobacter species in the civet cat gut may promote the observed increased

333 production of malic acid and citric acid in kopi luwak. Interestingly, the TCA cycle of

334 Gluconobacter spp. is incomplete due to the lack of succinate dehydrogenase for the

335 production of fumarate and succinate (Prust et al., 2005). As a result, the incomplete TCA cycle

336 may also help to boost the production of citric acid and malic acid.

337

338 Overall, our results reveal biological factors that may be involved in the fermentation of kopi

339 luwak in the gut of civet cat. Our current study focuses on only G. frateurii and G. japonicus, but

340 other civet cat fecal microbes, such as Citrobacter and Clostridium, may also help ferment kopi

341 luwak. To gain a more complete understanding of the biological factors involved in the

342 fermentation of kopi luwak in the civet cat gut, a metagenomic analysis should be performed to

343 investigate the functional genes that are highly expressed in most or all bacteria present in the

344 civet cat fecal microbiome.

345

\section{Conclusion}

347 In this study, we aimed to characterize the fecal microbiome of civet cats. We found, for the first

348 time, that the fecal microbiome of civet cats was dominated by Gluconobacter, namely $G$.

349 frateurii, G. japonicus, G. nephelii, and G. wancherniae, using the 16S rRNA gene sequence.

350 Moreover, we also identified Gluconobacter species as having the large number of genes

351 involved in cell motility and hydrogen sulfide and sulfur-containing amino acids, which may 
352 influence the fermentation of kopi luwak in the gut of civet cat.

353

354 Acknowledgments

355 We acknowledge funding from the Education Academy of Computational Life Sciences, Tokyo

356 Institute of Technology and from the Fundamental Research Grant Scheme (grant number:

357 MMUE.140076, Malaysia Ministry of Higher Education (MOHE)).

358

359 Figure Legends

360 Fig. 1. Hierarchical clustering of bacterial genera of microbes identified in feces from different

361 animals

362 The relative abundance of each bacterial genus was estimated for the fecal microbiome of

363 various animals. The microbial composition in feces from each animal is presented as the

364 relative abundance. The 10 most abundant genera observed in animal feces are labeled with

365 different colors; other genera are labeled "others". The hierarchical clustering tree was generated

366 based on the Euclidean distance between the bacterial genus level compositions.

367

368 Fig. 2 Phylogenetic tree for the Gluconobacter genus

369 The phylogenetic tree was based on 16S rRNA gene sequences (27F-338R) and was constructed

370 using the neighbor-joining method. The Acetobacter strains were used as an outgroup (green).

371 Data for Gluconobacter group 1 (orange) and group 2 (purple) were from the SILVA Living

372 Tree Project version 128. The Gluconobacter sequences identified in the civet cat fecal samples 
373 are clustered together (black) with group 2.

374

375 Fig. 3. KO terms of each Gluconobacter strain from the RefSeq database

376 Gluconobacter strains within each lineage had similar KO terms. Rows represent the 39

377 Gluconobacter strains, and columns represent the 1,653 KO terms that were identified in more

378 than one strain. The strains were clustered based on Euclidean distances calculated from the KO

379 terms for each Gluconobacter strain. The presence of a KO term is indicated by yellow

380 highlighting, and absences are indicated by black. Each Gluconobacter strain is colored based on

381 the phylogenetic analysis presented in Fig. 2, i.e., Gluconobacter group 1 is shown in orange,

382 Gluconobacter group 2 is shown in purple, and unknown species of Gluconobacter are not

383 colored.

384

385 Fig. 4. Hierarchical clustering of bacterial genera based on the number of paralogs for each $\mathrm{KO}$ 386 term

387 The Gluconobacter genus and species were clustered based on Euclidean distances calculated

388 from each median number of paralogs for each $\mathrm{KO}$ term $(3,627)$. The color scale of the heat-map 389 indicates the number of paralogs for each $\mathrm{KO}$ term.

390

391 Fig. 5. The characteristic KO terms for G. frateurii and G. japonicus in FuncTree2

392 The orange circle is the negative-log-scaled $P$ value obtained from Fisher's exact test. The green

393 bar is the positive ratio of G. frateurii and G. japonicus. The KO terms of the TCA cycle are

394 mapped at the top left of the figure. 


\section{Tables}

397 Table 1. A list of animals and their total number of the 16S rRNA gene DNA sequence samples 398 used in the comparative analysis.

\section{Supplemental Information}

400 Fig. S1. Multiple alignment results of the 16S rRNA sequences of Gluconobacter species $(G$.

401 cerinus, G. frateurii, G. japonicus, G. nephelii, G. thailandicus, and G. wancherniae)

402

403 Fig. S2. Maximum growth temperature of Gluconobacter species.

404

405 Table S1. The RefSeq genomes list of each bacterial genus used for comparative genomics

406

407 Table S2. Number of KO paralogs generated with the median of bacterial genus strains

Table S3. Microbial composition of each animal fecal sample

410

411 Table S4. Statistical value of the KO paralog numbers

412 


\section{References}

414 Agyirifo DS, Wamalwa M, Otwe EP, Galyuon I, Runo S, Takrama J, Ngeranwa J. 2019. Metagenomics

415 analysis of cocoa bean fermentation microbiome identifying species diversity and putative

416 functional capabilities. Heliyon 5:e02170.

417 Barberán A, Caceres Velazquez H, Jones S, Fierer N. 2017. Hiding in Plain Sight: Mining Bacterial

418 Species Records for Phenotypic Trait Information. mSphere 2. DOI: 10.1128/mSphere.00237-17.

419 Berean KJ, Ha N, Ou JZ, Chrimes AF, Grando D, Yao CK, Muir JG, Ward SA, Burgell RE, Gibson PR,

420 Kalantar-Zadeh K. 2018. The safety and sensitivity of a telemetric capsule to monitor

421 gastrointestinal hydrogen production in vivo in healthy subjects: a pilot trial comparison to

422 concurrent breath analysis. Alimentary pharmacology \& therapeutics 48:646-654.

423 Bolger AM, Lohse M, Usadel B. 2014. Trimmomatic: a flexible trimmer for Illumina sequence data.

424 Bioinformatics 30:2114-2120.

425 Buchfink B, Xie C, Huson DH. 2015. Fast and sensitive protein alignment using DIAMOND. Nature

426 methods 12:59-60.

427 Cullender TC, Chassaing B, Janzon A, Kumar K, Muller CE, Werner JJ, Angenent LT, Bell ME, Hay

428 AG, Peterson DA, Walter J, Vijay-Kumar M, Gewirtz AT, Ley RE. 2013. Innate and adaptive

429 immunity interact to quench microbiome flagellar motility in the gut. Cell host \& microbe 14:571-

$430 \quad 581$.

431 Czerny M, Mayer F, Grosch W. 1999. Sensory study on the character impact odorants of roasted arabica 432 coffee. Journal of agricultural and food chemistry 47:695-699.

433 De Bruyn F, Zhang SJ, Pothakos V, Torres J, Lambot C, Moroni AV, Callanan M, Sybesma W, Weckx S,

434 De Vuyst L. 2017. Exploring the Impacts of Postharvest Processing on the Microbiota and

435 Metabolite Profiles during Green Coffee Bean Production. Applied and environmental microbiology

436 83. DOI: $10.1128 /$ AEM.02398-16.

437 Deusch O, O’Flynn C, Colyer A, Morris P, Allaway D, Jones PG, Swanson KS. 2014. Deep Illumina- 
438

439

440

441

442

443

444

445

446

447

448

449

450

451

452

453

454

455

456

457

458

459

460

461

462

463

based shotgun sequencing reveals dietary effects on the structure and function of the fecal microbiome of growing kittens. PloS one 9:e101021.

Deusch O, O’Flynn C, Colyer A, Swanson KS, Allaway D, Morris P. 2015. A Longitudinal Study of the Feline Faecal Microbiome Identifies Changes into Early Adulthood Irrespective of Sexual Development. PloS one 10:e0144881.

Edgar RC. 2010. Search and clustering orders of magnitude faster than BLAST. Bioinformatics 26:2460 2461.

Ehrlich SD, Dusko Ehrlich S, The MetaHIT Consortium. 2011. MetaHIT: The European Union Project on Metagenomics of the Human Intestinal Tract. Metagenomics of the Human Body:307-316. DOI: 10.1007/978-1-4419-7089-3_15.

Fisher SRA. 1962. CONFIDENCE LIMITS FOR A CROSS-PRODUCT RATIO. The Australian journal of statistics 4:41-41.

de Jager V, Siezen RJ. 2011. Single-cell genomics: unravelling the genomes of unculturable microorganisms. Microbial biotechnology 4:431-437.

Johanna Haiko BW-W. 2013. The Role of the Bacterial Flagellum in Adhesion and Virulence. Biology $2: 1242$.

Jumhawan U, Putri SP, Yusianto, Bamba T, Fukusaki E. 2016. Quantification of coffee blends for authentication of Asian palm civet coffee (Kopi Luwak) via metabolomics: A proof of concept. Journal of bioscience and bioengineering 122:79-84.

Jumhawan U, Putri SP, Yusianto, Marwani E, Bamba T, Fukusaki E. 2013. Selection of discriminant markers for authentication of Asian palm civet coffee (Kopi Luwak): a metabolomics approach. Journal of agricultural and food chemistry 61:7994-8001.

Kanehisa M. 2000. KEGG: Kyoto Encyclopedia of Genes and Genomes. Nucleic acids research 28:2730.

Kartzinel TR, Hsing JC, Musili PM, Brown BRP, Pringle RM. 2019. Covariation of diet and gut microbiome in African megafauna. Proceedings of the National Academy of Sciences of the United 
States of America. DOI: 10.1073/pnas.1905666116.

465 Kumar S, Stecher G, Tamura K. 2016. MEGA7: Molecular Evolutionary Genetics Analysis Version 7.0 466 for Bigger Datasets. Molecular biology and evolution 33:1870-1874.

467 Lori MM, Claus WG. 1989. Phenotypic Characteristics Correlated with Deoxyribonucleic Acid Sequence 468 Similarities for Three Species of Gluconobacter: G. oxydans (Henneberg 1897) De Ley 1961, G.

469 frateurii sp. nov., and G. asaii sp. nov. International journal of systematic and evolutionary 470 microbiology 39:174-184.

471 Malimas T, Yukphan P, Takahashi M, Muramatsu Y, Kaneyasu M, Potacharoen W, Tanasupawat S, 472 Nakagawa Y, Tanticharoen M, Yamada Y. 2009. Gluconobacter japonicus sp. nov., an acetic acid bacterium in the Alphaproteobacteria. International journal of systematic and evolutionary microbiology 59:466-471.

Marcone M. 2004. Composition and properties of Indonesian palm civet coffee (Kopi Luwak) and Ethiopian civet coffee. Food research international 37:901-912.

MicrobeDB.jp Project Team. 2017.Metagenome and Microbes Environmental Ontology. Available at https://bioportal.bioontology.org/ontologies/MEO (accessed June 19, 2018).

Moeller AH, Li Y, Mpoudi Ngole E, Ahuka-Mundeke S, Lonsdorf EV, Pusey AE, Peeters M, Hahn BH, Ochman H. 2014. Rapid changes in the gut microbiome during human evolution. Proceedings of the National Academy of Sciences of the United States of America 111:16431-16435.

Mori H, Maruyama T, Yano M, Yamada T, Kurokawa K. 2018. VITCOMIC2: visualization tool for the phylogenetic composition of microbial communities based on 16S rRNA gene amplicons and metagenomic shotgun sequencing. BMC systems biology 12:30.

Nakamura T, Yamada KD, Tomii K, Katoh K. 2018. Parallelization of MAFFT for large-scale multiple sequence alignments. Bioinformatics . DOI: 10.1093/bioinformatics/bty121.

487 NITE. 2018.Growth temperature condition of Gluconobacter. Available at 488 http://www.nite.go.jp/nbrc/cultures/support/gluconobacter.html (accessed March 21, 2018).

489 Nyakatura K, Bininda-Emonds ORP. 2012. Updating the evolutionary history of Carnivora (Mammalia): 
a new species-level supertree complete with divergence time estimates. BMC biology 10:12.

491 Patou M-L, Wilting A, Gaubert P, Esselstyn JA, Cruaud C, Jennings AP, Fickel J, Veron G. 2010.

492 Evolutionary history of the Paradoxurus palm civets - a new model for Asian biogeography. Journal

$493 \quad$ of biogeography 37:2077-2097.

494 Prust C, Hoffmeister M, Liesegang H, Wiezer A, Fricke WF, Ehrenreich A, Gottschalk G, Deppenmeier

495 U. 2005. Complete genome sequence of the acetic acid bacterium Gluconobacter oxydans. Nature

496 biotechnology 23:195-200.

497 R Core Team.R: The R Project for Statistical Computing. Available at https://www.R-project.org/

$498 \quad$ (accessed June 3, 2019).

499 Reineccius G. 2005. Flavor Chemistry and Technology, Second Edition.

500 Suhandono S, Setiadi H, Kristianti T, Kusuma AB, Wedaringtyas AW, Djajadi DT, Nyoman Pugeg

501 Aryantha I. 2016. Diversity of Culturable Bacterial in Various Parts of Luwak's (Paradoxurus

502 hermaprodithus javanica) Gastrointestinal Tract. Microbiology Indonesia 10:65-70.

503 Turnbaugh PJ, Ley RE, Hamady M, Fraser-Liggett CM, Knight R, Gordon JI. 2007. The Human

504 Microbiome Project. Nature 449:804-810. DOI: 10.1038/nature06244.

505 Uchiyama T, Irie M, Mori H, Kurokawa K, Yamada T. 2015. FuncTree: Functional Analysis and

$506 \quad$ Visualization for Large-Scale Omics Data. PloS one 10:e126967.

507 Yilmaz P, Parfrey LW, Yarza P, Gerken J, Pruesse E, Quast C, Schweer T, Peplies J, Ludwig W,

508 Glöckner FO. 2014. The SILVA and "All-species Living Tree Project (LTP)" taxonomic

509 frameworks. Nucleic acids research 42:D643-8.

510

Peer] reviewing PDF | (2020:01:45194:1:1:REVIEW 13 Jun 2020) 


\section{Figure 1}

Hierarchical clustering of bacterial genera of microbes identified in feces from different animals

The relative abundance of each bacterial genus was estimated for the fecal microbiome of various animals. The microbial composition in feces from each animal is presented as the relative abundance. The 10 most abundant genera observed in animal feces are labeled with different colors; other genera are labeled "others". The hierarchical clustering tree was generated based on the Euclidean distance between the bacterial genus level compositions.

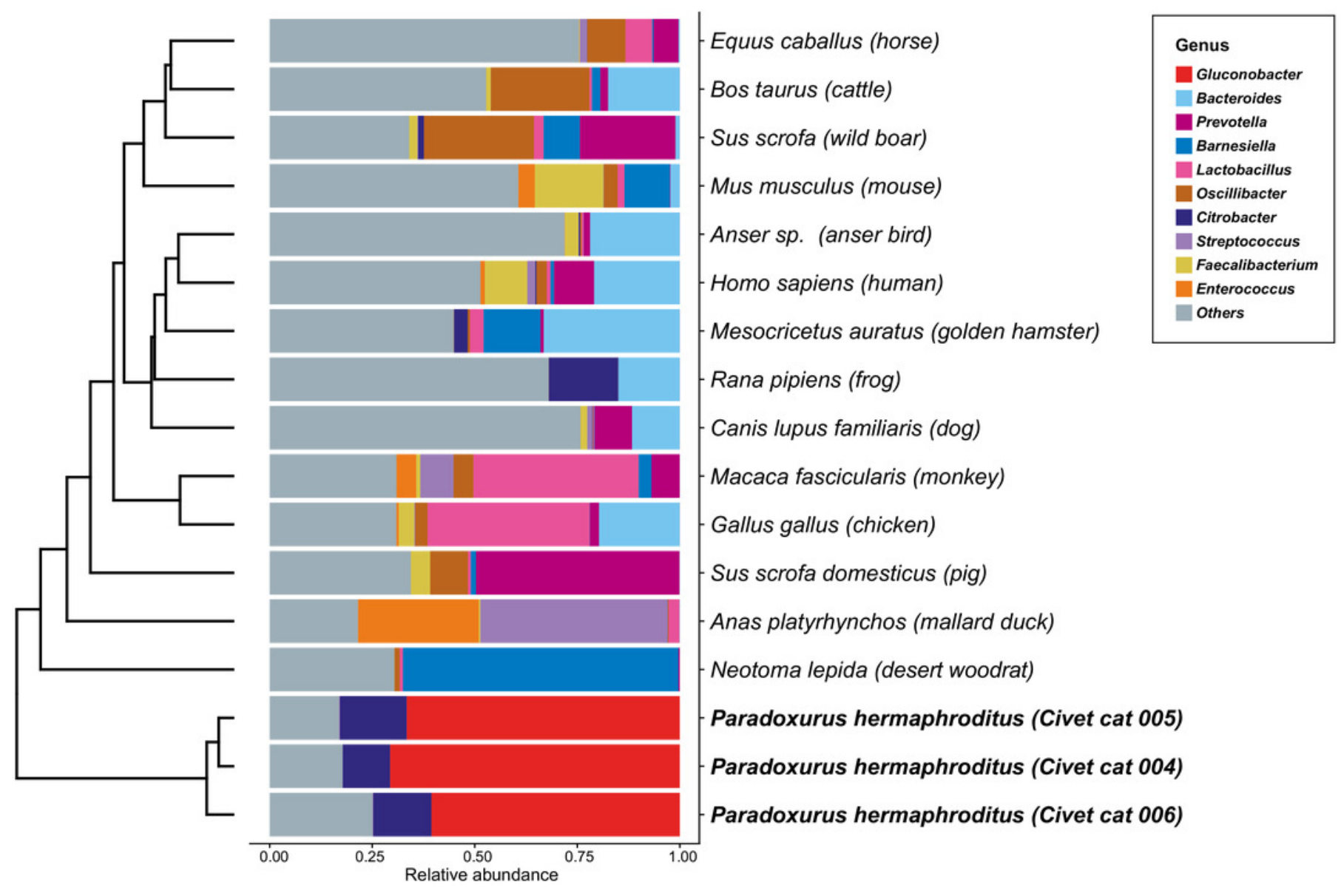




\section{Figure 2}

Phylogenetic tree for the Gluconobacter genus

The phylogenetic tree was based on 16S rRNA gene sequences (27F-338R) and was constructed using the neighbor-joining method. The Acetobacter strains were used as an outgroup (green). Data for Gluconobacter group 1 (orange) and group 2 (purple) were from the SILVA Living Tree Project version 128. The Gluconobacter sequences identified in the civet cat fecal samples are clustered together (black) with group 2.

Gluconobacter sequence of civet cat fecal samples
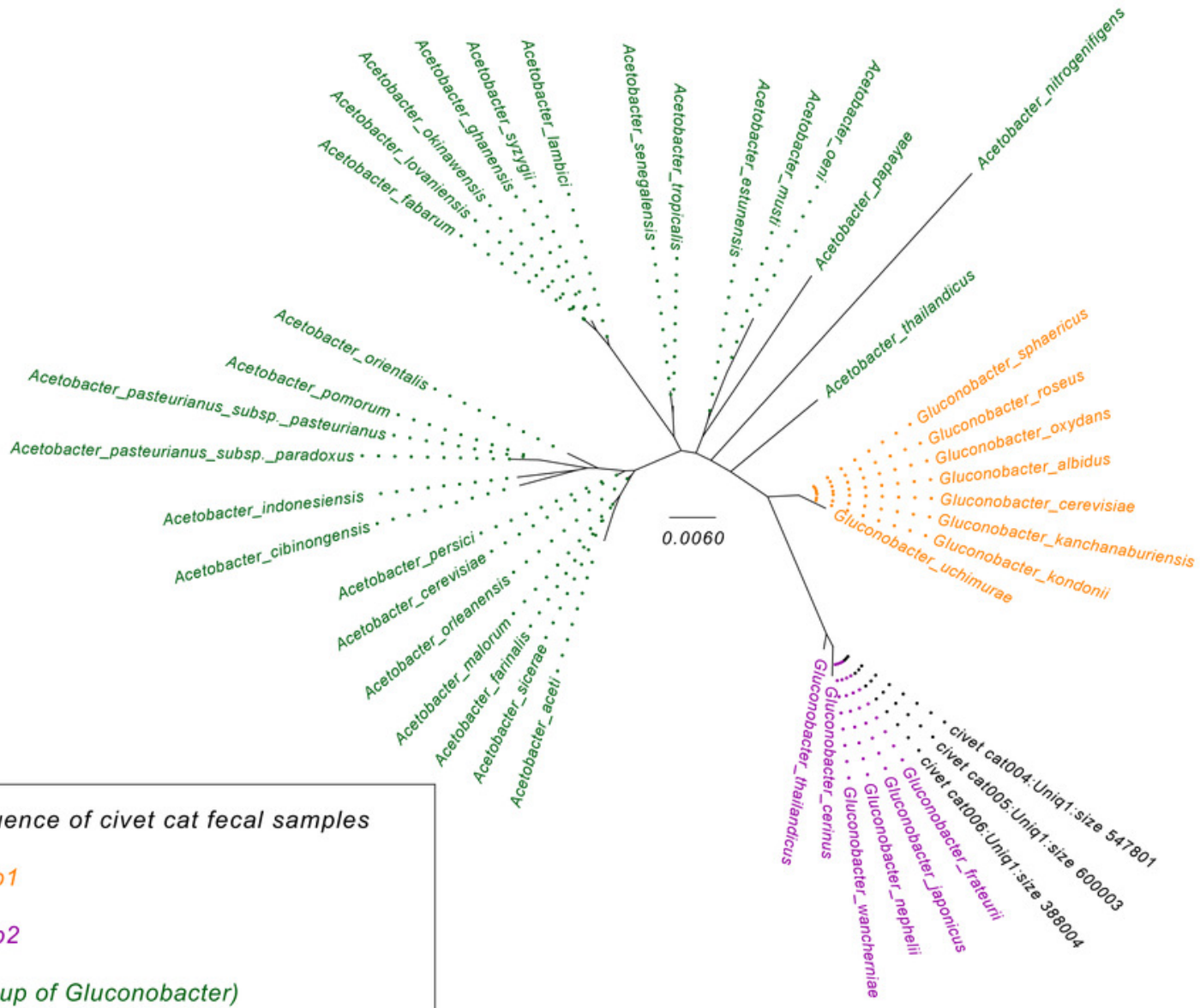


\section{Figure 3}

\section{KO terms of each Gluconobacter strain from the RefSeq database}

Gluconobacter strains within each lineage had similar KO terms. Rows represent the 39

Gluconobacter strains, and columns represent the 1,653 KO terms that were identified in more than one strain. The strains were clustered based on Euclidean distances calculated from the KO terms for each Gluconobacter strain. The presence of a KO term is indicated by yellow highlighting, and absences are indicated by black. Each Gluconobacter strain is colored based on the phylogenetic analysis presented in Fig. 2, i.e., Gluconobacter group 1 is shown in orange, Gluconobacter group 2 is shown in purple, and unknown species of Gluconobacter are not colored.
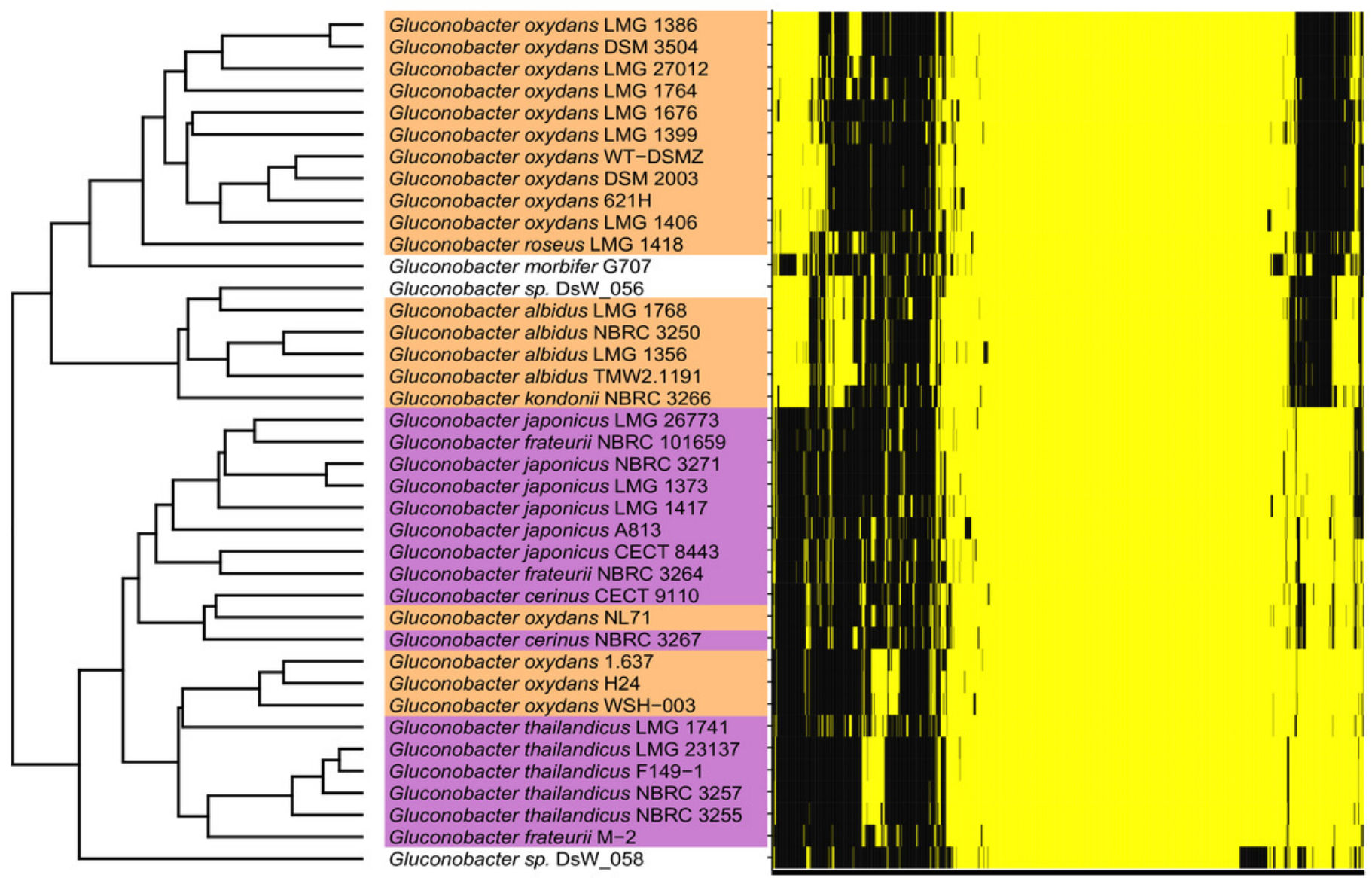

KO 
Figure 4

Figure 4. Hierarchical clustering of bacterial genera based on the number of paralogs for each KO term

The genus and Gluconobacter species were clustered based on Euclidean distances calculated from each median number of paralogs for each of the KO terms $(3,627)$. The color scale of the heat-map indicates the number of paralogs for each KO term.
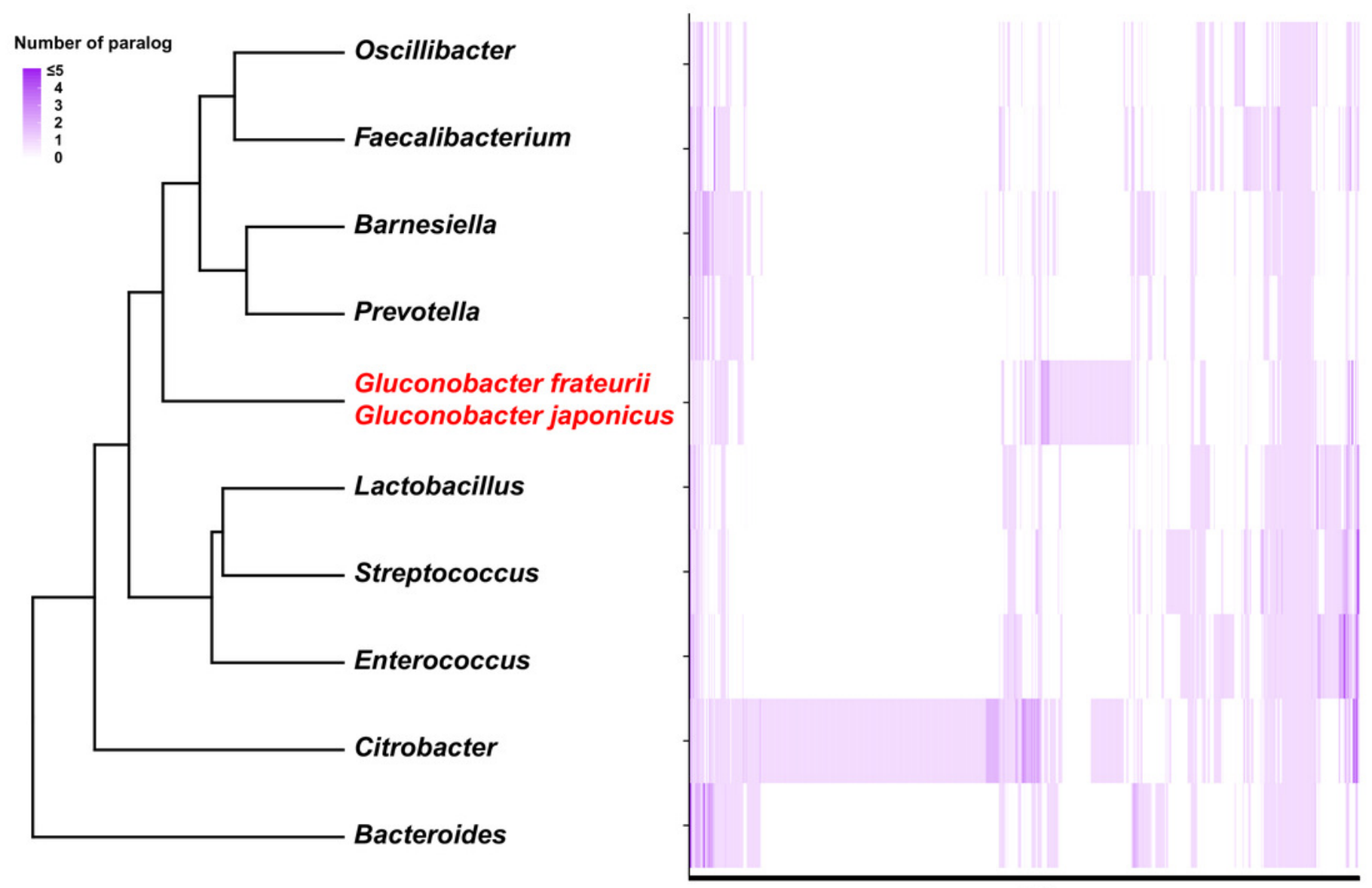
Figure 5

The characteristic KO terms for $G$. frateurii and G. japonicus in FuncTree2

The orange circle is the negative-log-scaled $P$ value obtained from Fisher's exact test. The green bar is the positive ratio of $G$. frateurii and $G$. japonicus. The KO terms of the TCA cycle are mapped at the top left of the figure. 

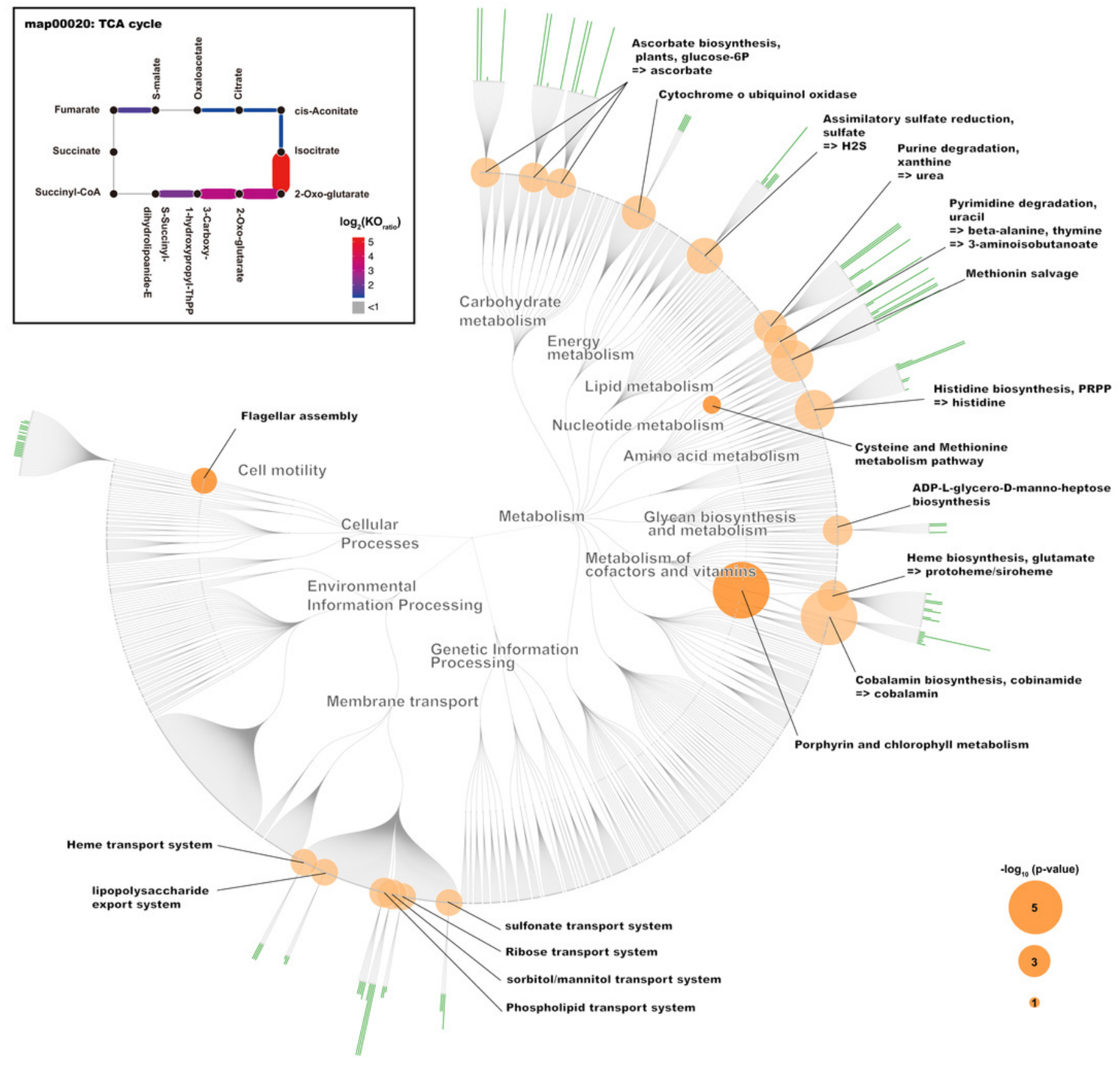

$-\log _{10}(\mathrm{p}$-value $)$ 


\section{Table $\mathbf{1}$ (on next page)}

A list of animals and their total number of the 16S rRNA gene DNA sequence samples used in the comparative analysis. 


\begin{tabular}{|c|c|c|c|}
\hline Taxonomy & Animal & Number of fecal sample & Collected from \\
\hline Paradoxurus hermaphroditus & civet cat & 3 & This study \\
\hline Anas platyrhynchos & mallard duck & 1 & MicrobeDB.jp \\
\hline Anser $s p$ & anser bird & 1 & MicrobeDB.jp \\
\hline Bos taurus & cattle & 2 & MicrobeDB.jp \\
\hline Canis lupus familiaris & $\operatorname{dog}$ & 69 & MicrobeDB.jp \\
\hline Equus caballus & horse & 166 & MicrobeDB.jp \\
\hline Gallus gallus & chicken & 1 & MicrobeDB.jp \\
\hline Homo sapiens & human & 3003 & MicrobeDB.jp \\
\hline Macaca fascicularis & monkey & 353 & MicrobeDB.jp \\
\hline Mesocricetus auratus & golden hamster & 29 & MicrobeDB.jp \\
\hline Mus musculus & mouse & 1346 & MicrobeDB.jp \\
\hline Neotoma lepida & desert woodrat & 22 & MicrobeDB.jp \\
\hline Rana pipiens & frog & 9 & MicrobeDB.jp \\
\hline Sus scrofa & wild boar & 1 & MicrobeDB.jp \\
\hline Sus scrofa domesticus & pig & 141 & MicrobeDB.jp \\
\hline
\end{tabular}

